\title{
The relationship between non-alcoholic fatty liver disease and breast cancer: a retrospective case-control study
}

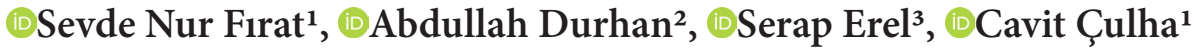 \\ ${ }^{1}$ University of Health Sciences, Ankara Training and Research Hospital, Department of Endocrinology and Metabolism, Ankara, Turkey \\ ${ }^{2}$ University of Health Sciences, Ankara Training and Research Hospital, Department of Surgical Oncology, Ankara, Turkey \\ ${ }^{3}$ University of Health Sciences, Ankara Training and Research Hospital, Department of General Surgery, Ankara, Turkey
}

Cite this article as: Fırat SN, Durhan A, Erel S, Çulha C. The relationship between non-alcoholic fatty liver disease and breast cancer: a retrospective case-control study. J Health Sci Med 2022; 5(1): 109-113.

\begin{abstract}
Aim: Breast cancer is the most common cancer among women and is one of the main causes of death in women. It is known that some metabolic components that are risk factors for non-alcoholic fatty liver disease are also risk factors for breast cancer. The aim of this retrospective cohort study was to show the prevalence of non-alcoholic fatty liver disease breast cancer patients and the effect of non-alcoholic fatty liver disease on breast cancer development through comparisons with a control group with normal mammography.
\end{abstract}

Material and Method: The study included 108 patients who were operated on for breast cancer in the general surgery clinic of our hospital between January 2015 and December 2018 and who underwent abdominal ultrasound for breast cancer staging. A control group was formed of 102 women with benign breast lesions on routine mammography and who underwent abdominal ultrasound within 6 months before mammography. The prevalence of diabetes, hypertension and hyperlipidemia and body mass indexes were similar in both groups.

Results: In the evaluation of all the study participants, an association was found between non-alcoholic fatty liver disease and breast cancer. In the obese and non-obese subgroups, non-alcoholic fatty liver disease was significantly associated with breast cancer in the non-obese subgroup. [OR 2.67\%, 95\% confidence interval [95\% CI) 1.1-6.0, p=0.020].

Conclusion: Non-alcoholic fatty liver disease was seen to be significantly associated with breast cancer regardless of known risk factors. This relationship there was in non-obese women with NAFLD, but not in the obese group.

Keywords: Breast cancer, nonalcoholic fatty liver disease, non-obese women, obesity

\section{INTRODUCTION}

Breast cancer is important because it is the most common cancer type in female population and one of the main causes of deaths associated with cancer among women (1). Late age in menopause, early menarche, hormonal or reproductive factors, family history, late pregnancy, and nulliparity are certain risk factors for this disease $(2,3)$. Recent meta-analyses have also shown an association of metabolic syndrome components and obesity with an increased breast cancer risk (4).

There is an association between non-alcoholic fatty liver disease (NAFLD) and such factors as metabolic abnormalities and obesity. NAFLD is an increasingly widespread clinical condition and an important health problem because it may progress to cirrhosis, non- alcoholic steatohepatitis (NASH), hepatocellular carcinoma, and end-stage liver disease. Recent studies have shown that NAFLD may be an additional risk factor for non-hepatic cancers in the gastrointestinal tract $(5,6)$. However, there are few studies in the literature on the relationship of NAFLD with breast cancer $(7,8)$.

Mammography is the most effective method for breast cancer screening. Liver biopsy is known as the gold standard for NAFLD diagnosis; however, its use in clinical practice is limited since it is an invasive test. Ultrasound is an accurate imaging method, which is used to help detect and characterize hepatic steatosis (9).

This study is aimed at determining the relationship of NAFLD with breast cancer. 


\section{MATERIAL AND METHOD}

The study was carried out with the permission of University of Health Sciences, Ankara Training and Research Hospital Clinical Researchs Ethics Committee (Date: 17.09.2020, Decision No: 20-367). All procedures were carried out in accordance with the ethical rules and the principles of the Declaration of Helsinki.

The study included the data obtained from 108 breast cancer patients who were operated on in the Department of General Surgery between January 2015 and September 2018. A control group was formed of 102 women with benign breast masses detected on mammography. The study exclusion criteria included having autoimmune hepatitis, chronic viral hepatitis $\mathrm{B} / \mathrm{C}$, significant alcohol consumption (140 g/week) and other chronic liver diseases. A record was made for each patient of the clinical, anthropometric, radiological and laboratory data. The following formula was utilized to calculate the BMI of the patients: weight $(\mathrm{kg} /$ height square $\mathrm{m} 2)$. Those with BMI $\geq 30$ were considered obese. Laboratory studies included alanine aminotransferase (ALT), fasting blood glucose, and serum aspartate aminotransferase (AST). Patients with $>3$-fold the upper limit of normal for AST and/or ALT were defined as liver damage and these patients were also excluded. Molecular subtypes of breast cancer (luminal A, luminal B, HER2 +, and triple negative), tumor size, and Ki-67 indices were obtained from the hospital database. Breast cancer subtypes were grouped as follows; Luminal A; Patients with positive/ exhibiting ER and PR receptors, Luminal B; It includes $\mathrm{ER}$ and $\mathrm{PR}$ receptors positive/exhibiting and higher histological grade than Luminal A, triple negative (ER, PR and HER-2 negative/non-exhibiting) group.

Fatty liver disease was evaluated according to the reports of abdominal ultrasonography performed for staging when breast cancer was diagnosed. For control subjects with benign breast masses, the abdominal ultrasonography reports taken within six months of the mammography were recorded. The mammographies were evaluated in line with the Breast Imaging Reporting and Data System (BI-RADS). According to this tool, category-1 includes negative and category-2 benign findings. Possibly benign findings are included in category-3. Findings in category-4 are labeled as suspicious for malignancy. Finally, category- 5 is for findings that are highly significant malignancy10. Patients with BI-RADS 1,2 , and 3 mammographies were included in the control group.

The study data were statistically analyzed through SPSS version 21. The Kolmogorov-Smirnov and Shapiro-Wilk tests were used in order to assess conformity of the data to normal distribution. Descriptive statistics for continuous variables were stated as mean \pm standard deviation and minimum-maximum values. The statistical significance of the difference in categorical variables between the study groups was evaluated with the Chi-square test. The mean differences between the groups were compared with One-Way ANOVA. When parametric test assumptions were not met, the comparison of both ordered data and continuous variables was performed using the MannWhitney $U$ test. Finally, multivariate logistic regression analysis was used to analyze how fatty liver affected breast cancer. All tests applied had two tails; moreover, statistical significance was designated as $\mathrm{p}<0.05$.

\section{RESULTS}

Of the 210 patients included in the study, benign breast disease was determined in 102 (48.5\%) with a mean age of $54.5 \pm 11.6$ years, and breast cancer was determined in $108(51.50 \%)$ with a mean age of $52.4 \pm 10.1$ years. Table 1 shows the clinical and demographic characteristics of the two groups. Age, BMI, the prevalence of HT, DM, HL and AST, ALT and glucose levels were similar in both groups.

\begin{tabular}{|c|c|c|c|}
\hline Characteristics & $\begin{array}{l}\text { Control } \\
(\mathrm{n}: 102)\end{array}$ & $\begin{array}{c}\text { Breast cancer } \\
(\mathrm{n}: 108)\end{array}$ & $\underset{\text { value }}{\mathbf{p}}$ \\
\hline Age, mean, years & $52.4( \pm 10.1)$ & $54.5( \pm 11.6)$ & 0.157 \\
\hline BMI, mean, $(\mathrm{kg} / \mathrm{m} 2)$ & $29.6( \pm 4.8)$ & $29.2( \pm 4.8)$ & 0.845 \\
\hline $\mathrm{DM}, \mathrm{n}(\%)$ & $21(20.5)$ & $18(16.6)$ & 0.465 \\
\hline HT, n (\%) & $22(21.5)$ & $22(20.3)$ & 0.831 \\
\hline $\mathrm{HL}, \mathrm{n}(\%)$ & $13(12.7)$ & $11(10.1)$ & 0.560 \\
\hline NAFLD, n (\%) & $30(29.4)$ & $48(44.4)$ & $0.025^{*}$ \\
\hline Glucose, mean $\pm \mathrm{SD},(\mathrm{mg} / \mathrm{dl})$ & $106( \pm 30.9)$ & $103.1( \pm 27.3)$ & 0.224 \\
\hline ALT (IU/L), mean & $17.8( \pm 7.2)$ & $18.1( \pm 9.1)$ & 0.891 \\
\hline AST(IU/L), mean & $19.2( \pm 6.8)$ & $18.8( \pm 5.8)$ & 0.899 \\
\hline
\end{tabular}

In the breast cancer group, NAFLD prevalence was $44.4 \%$ (48/108), which was significantly higher than the control $(29.4 \%, 30 / 102)(\mathrm{p}=0.025)$. The subgroup analysis revealed that fatty liver was linked with breast cancer occurrence in the non-obese group, but NAFLD and breast cancer were not associated in the obese group ( $\mathrm{p}=0.014, \mathrm{p}=0.336$, respectively). Multivariate analysis showed that in the total study population a significant association existed between NAFLD and breast cancer [odds ratio (OR) 1.92\%, 95\% confidence interval (95\%CI) 1.0-3.3, $(\mathrm{p}=0.025)]$. When the patients were evaluated as obese and non-obese, NAFLD was found to be statistically significantly related to breast cancer in the non-obese subgroup [OR 2.67\%, 95\% confidence interval (95\%CI) 1.1-6.0, $\mathrm{p}=0.020$ ] (Table 2). No significant association was revealed in the obese group $(\mathrm{p}=0.337)$ between NAFLD and breast cancer. 


\begin{tabular}{|llc|}
\hline \multicolumn{3}{|l|}{ Table 2. Results of the multivariate analysis } \\
\hline \multicolumn{4}{|c|}{ OR (95\% CI) } & p value \\
\hline Total population NAFLD & $1.92(1.0-3.3)$ & $0.025^{*}$ \\
Nonobese group NAFLD & $2.67(1.1-6.0)$ & $0.020^{*}$ \\
\hline NAFLD: Non-alcoholic fatty liver disease \\
\hline
\end{tabular}

Invasive ductal carcinoma (85.2\%) was the most common pathological subtype in breast cancer patients. Tumor diameter was approximately $2.5 \mathrm{~cm}$. There was lymph node metastasis in $38 \%$ of the patients and the tumor was unifocal in $86.1 \%$. The mean value of the Ki- 67 index was $15.09 \pm 12.9$.

In the molecular subtype staging of the patients, 39(36.1\%) were luminal A, 32 (29.6\%) were luminal B, 36(33.3\%) were HER-2 positive, and $1(0.9 \%)$ was triple negative. The evaluation of all the patients could not find out any statistically significant differences between the presence of NAFLD and molecular subtypes. In the evaluation of the subgroup of obese patients, the presence of NAFLD was higher in the luminal B group than the luminal A group, which was statistically significant $(\mathrm{p}=0.042)$ (Table 3). Also, at the end of the evaluation of the relationships between NAFLD and histological subtype of breast cancer, there were no significant differences between the presence of NAFLD and histological subtype. There was no significant relationship between Ki-67 indexes and NAFLD.

In the control group, 4 (3.9\%) patients were BI-RADS-1, 46 (45.1\%) were BI-RADS-2, and $52(51 \%)$ were BIRADS-3. No significant association was detected between the BI-RADS score of the patients and NAFLD $(\mathrm{p}=0.316)$.

\section{DISCUSSION}

According to the results of this study, which compared the relationship of NAFLD with breast cancer in patients and controls, NAFLD is related to breast cancer independently of known risk factors. Moreover, there was a relationship between the presence of NAFLD and breast cancer in the non-obese subgroup.
Despite the widespread use of breast cancer screening methods and developing technology, it is still the most frequent type of cancer and the leading death cause for women. Most commonly known controllable risk factors for breast cancer include genetics, diet, lifestyle, hormone replacement therapy, alcohol consumption and obesity $(1,11,12)$. Based on these factors, there are numerous studies showing that breast cancer is associated with obesity, metabolic syndrome, and diabetes $(6,13)$. Moreover, these factors are also known risk factors for NAFLD. Dyslipidemia, sleep apnea, family history, hypothyroidism, hypogonadism, and sedentary lifestyle are other factors in NAFLD etiology (14). The common metabolic risk factors of breast cancer and NAFLD suggest a potential relationship between these two diseases. Another important factor in NAFLD physiopathology is insulin resistance due to obesity. Excessive fatty acids accumulating in the cell with insulin resistance cause an increase in oxidative stress parameters and a decrease in antioxidants. This situation causes mitochondrial dysfunction, uncontrolled cytokine release, especially TNF-alpha, increased IL-8 and necroinflammation. There is a decrease in adiponectin, which is known to be an anti-inflammatory, anti-TNF and liver-protective cytokine. Decreased adiponectin levels lead to significant insulin resistance, followed by increased insulin levels and growth factor-1 (IGF-1), which bind to its receptors in the cell, causing cell proliferation and increased vascular endothelial growth factor production $(15,16)$. Another reason is the high leptin levels in the blood, which both increase insulin resistance and affect the development of carcinogenesis. In recent years, many studies have been conducted on different types of cancer related to inflammation and cancer development $(17,18)$.

NAFLD is known to cause liver, heart and kidney diseases, and many studies on the risk of extrahepatic malignancy have reported a relationship between NAFLD and some types of cancer $(19,20)$. However, there are few studies in the literature on the association of NAFLD with breast cancer. Kwak et al. (8) reported a statistically significant difference in NAFLD between non-obese breast cancer patients and a control group, similar to our study. Nseir et

\begin{tabular}{|c|c|c|c|c|c|}
\hline & Luminal A & Luminal B & HER-2 positive & Triple negative & p value \\
\hline \multicolumn{6}{|l|}{ Total breast cancer } \\
\hline NAFLD, n (\%) & $15(31.2)$ & $16(33.3)$ & $17(35.4)$ & - & \\
\hline No NAFLD, n (\%) & $24(40)$ & $16(26.6)$ & $19(31.6)$ & $1(1.66)$ & 0.598 \\
\hline \multicolumn{6}{|l|}{ Obese group } \\
\hline NAFLD n (\%) & $7(21.2)^{a}$ & $12(36.3)^{a}$ & $4(12.1)$ & & \\
\hline No NAFLD, n (\%) & $11(55)$ & $3(15)$ & $6(30)$ & & $0.042^{*}$ \\
\hline \multicolumn{6}{|l|}{ Nonobese group } \\
\hline NAFLD, n (\%) & $8(32)$ & $4(16)$ & $13(52)$ & - & \\
\hline No NAFLD, n (\%) & $13(32.5)$ & $13(32.5)$ & $13(32.5)$ & $1(2.5)$ & 0.304 \\
\hline
\end{tabular}


al. (7) showed that NAFLD and estrogen use are predictive factors for breast cancer. Lee et al. (21) also showed that NAFLD is a predictive factor in terms of breast cancer and a prognostic factor for its recurrence. In our current study, when breast cancer and benign breast patients were compared, NAFLD was found to be statistically higher in breast cancer patients $(44.4 \%$ vs $29.4 \%)(p=0.024)$. In addition, when divided into subgroups, NAFLD was found to be statistically considerably higher among patients with breast cancer within the non-obese group. This particular finding is important because it shows that NAFLD may be associated with breast cancer independently of obesity. The current study results were not statistically significant when breast cancer cases were compared according to hormone receptor positivity and ki-67 index to define the mechanical relationship with NAFLD. Similar to the current study, Kwak et al. reported no significant relationship between hormone receptor positivity and NAFLD (8).

Liver biopsy constitutes the gold standard for NAFLD diagnosis, but since it is an invasive test and cannot be routinely applied to every patient, the patients selected for this study were those with hepatobiliary ultrasound, as a non-invasive imaging method (22). In literature, ultrasonography and tomography examinations have been mostly preferred $(7,8,23)$. NAFLD may be a risk factor for cancer disease or may develop depending on treatment such as chemotherapy and endocrine therapy. NAFLD has been described in case reports of patients with breast cancer due to tamoxifen (24). Tamoxifen leads to hepatic fatty liver by raising serum triglycerides, activating the b-oxidation pathway, and suppressing the synthesis of estrogen (25). Therefore, breast cancer patients with NAFLD who are receiving endocrine therapy should be followed closely due to the risk of recurrence and increased morbidity.

This study was a single-centered, retrospective one with a small sample size of 210 patients, which were the main limitations. Our study was retrospective, therefore, it is difficult to say that the presence of NAFLD is a independent risk factor for the development of breast cancer. The diagnosis of NAFLD was made with USG, which is a non-invasive imaging method, rather than biopsy. It can be recommended that future studies could use magnetic resonance imaging, which is a non-invasive test with high sensitivity and specificity.

\section{CONCLUSION}

In this study, an association was found between NAFLD and breast cancer. This association was also present in the non-obese population with NAFLD. These results are important in showing that the may be associated with the development of breast cancer in both obese and nonobese women with NAFLD.

\section{ETHICAL DECLARATIONS}

Ethics Committee Approval: The study was carried out with the permission of University of Health Sciences, Ankara Training and Research Hospital Clinical Researchs Ethics Committee (Date: 17.09.2020, Decision No: 20-367).

Informed Consent: Because the study was designed retrospectively, no written informed consent form was obtained from patients.

Referee Evaluation Process: Externally peer-reviewed.

Conflict of Interest Statement: The authors have no conflicts of interest to declare.

Financial Disclosure: The authors declared that this study has received no financial support.

Author Contributions: All of the authors declare that they have all participated in the design, execution, and analysis of the paper and that they have approved the final version

Note: This article presented at congresses as an oral presentation at the " 3 th National Breast Surgery Congress" on May 28-30, 2021.

\section{REFERENCES}

1. Ferlay J, Soerjomataram I, Dikshit R, et al. Cancer incidence and mortality worldwide: sources, methods and major patterns in GLOBOCAN 2012. Int J Cancer 2015; 136: 359-86.

2. Suh JS, Yoo KY, Kwon OJ, et al. Menstrual and reproductive factors related to the risk of breast cancer in Korea. Ovarian hormone effect on breast cancer. J Korean Med Sci 1996; 11: 501-8.

3. Nelson HD, Zakher B, Cantor A, et al. Risk factors for breast cancer for women aged 40 to 49 years: a systematic review and meta-analysis. Annals of Internal Medicine 2012; 156: 635-48.

4. Bhandari R, Kelley GA, Hartley TA, Rockett IR. Metabolic syndrome is associated with increased breast cancer risk: a systematic review with meta-analysis. Int J Breast Cancer 2014; 2014: 189384.

5. Tilg H, Diehl AM. NAFLD and extrahepatic cancers: have a look at the colon. Gut 2011; 60: 745-6.

6. Tsilidis KK, Kasimis JC, Lopez DS, Ntzani EE, Ioannidis JP. Type 2 diabetes and cancer: umbrella review of meta-analyses of observational studies. BMJ (Clinical research ed) 2015; 350: g7607.

7. Nseir W, Abu-Rahmeh Z, Tsipis A, Mograbi J, Mahamid M. Relationship between non-alcoholic fatty liver disease and breast cancer. The Israel Medical Association J: IMAJ 2017; 19: 242-5.

8. Kwak MS, Yim JY, Yi A, et al. Nonalcoholic fatty liver disease is associated with breast cancer in nonobese women. Dig Liver Dis 2019; 51: 1030-5.

9. Rinella ME. Nonalcoholic fatty liver disease: a systematic review. Jama 2015; 313: 2263-73.

10.D’Orsi CJ SE, Mendelson EB, Morris EA, et al. ACR BI-RADS ${ }^{\curvearrowleft}$ Atlas, Breast Imaging Reporting and Data System. Reston, VA, American College of Radiology; 2013.

11.Smith-Warner SA, Spiegelman D, Yaun SS, et al. Intake of fruits and vegetables and risk of breast cancer: a pooled analysis of cohort studies. Jama 2001; 285: 769-76.

12.Smith-Warner SA, Spiegelman D, Yaun SS, et al. Alcohol and breast cancer in women: a pooled analysis of cohort studies. Jama 1998; 279: 535-40. 
13. Rosato V, Bosetti C, Talamini R, et al. Metabolic syndrome and the risk of breast cancer in postmenopausal women. Ann Oncol 2011; 22: 2687-92.

14. Souza MR, Diniz Mde F, Medeiros-Filho JE, Araújo MS. Metabolic syndrome and risk factors for non-alcoholic fatty liver disease. Arq Gastroenterol 2012; 49: 89-96.

15. Cave M, Deaciuc I, Mendez C, et al. Nonalcoholic fatty liver disease: predisposing factors and the role of nutrition. J Nutr Biochem 2007; 18: 184-95.

16. Shoelson SE, Lee J, Goldfine AB. Inflammation and insulin resistance. J Clin Invest 2006; 116: 1793-801.

17. Hu B, Yang XR, Xu Y, et al. Systemic immune-inflammation index predicts prognosis of patients after curative resection for hepatocellular carcinoma. Clin Cancer Res 2014; 20: 6212-22.

18. Roxburgh CS, McMillan DC. Role of systemic inflammatory response in predicting survival in patients with primary operable cancer. Future Oncol 2010; 6: 149-63.

19.Sanna C, Rosso C, Marietti M, Bugianesi E. Non-alcoholic fatty liver disease and extra-hepatic cancers. Int J Mol Sci 2016; 17: 717.

20. Byrne CD, Targher G. NAFLD: a multisystem disease. J Hepatol 2015; 62: 47-64.

21.Lee YS, Lee HS, Chang SW, et al. Underlying nonalcoholic fatty liver disease is a significant factor for breast cancer recurrence after curative surgery. Medicine 2019; 98: e17277.

22. Sumida Y, Nakajima A, Itoh Y. Limitations of liver biopsy and noninvasive diagnostic tests for the diagnosis of nonalcoholic fatty liver disease/nonalcoholic steatohepatitis. World J Gastroenterol 2014; 20: 475-85.

23. Lee SS, Park SH. Radiologic evaluation of nonalcoholic fatty liver disease. World J Gastroenterol 2014; 20: 7392-402.

24. Pinto HC, Baptista A, Camilo ME, de Costa EB, Valente A, de Moura MC. Tamoxifen-associated steatohepatitis--report of three cases. J Hepatol 1995; 23: 95-7.

25. Yang YJ, Kim KM, An JH, et al. Clinical significance of fatty liver disease induced by tamoxifen and toremifene in breast cancer patients. Breast 2016; 28: 67-72. 